\title{
MicroRNA-106b functions as an oncogene and regulates tumor viability and metastasis by targeting LARP4B in prostate cancer
}

\author{
WEIQI YIN, JUNFENG CHEN, GUOYAO WANG and DONGXU ZHANG \\ Department of Urology, Ningbo First Hospital, Ningbo, Zhejiang 315010, P.R. China
}

Received September 10, 2018; Accepted March 7, 2019

DOI: $10.3892 / \mathrm{mmr} .2019 .10343$

\begin{abstract}
Prostate cancer (PCa) is the most common malignancy among males worldwide, and is one of the leading causes of cancer-related mortality. MicroRNAs (miRs) are a type of endogenous, noncoding RNA that serve a key role in pathological processes, and have been demonstrated to be involved in the formation and progression of $\mathrm{PCa}$. Previous studies have reported that miR-106b acts as an oncogene; however, the specific effects of miR-106b on PCa have not been fully elucidated. The present study aimed to investigate the role and underlying molecular mechanisms of miR-106b in the initiation and progression of PCa. In this study, miR-106b was reported to be overexpressed and la-related protein 4B (LARP4B) was downregulated in $\mathrm{PCa}$ tissues compared with paracancerous tissues. In addition, LARP4B was identified as a target gene of miR-106b by bioinformatics prediction analysis and a dual luciferase reporter gene assay. Furthermore, MTT, wound healing and Transwell assays were performed to evaluate PCa cell viability, and migration and invasive abilities. The data revealed that inhibition of miR-106b significantly suppressed the viability, migration and invasion of PCa cells. In addition, inhibition of miR-106b significantly suppressed the mRNA and protein expression of cancer-related genes, including matrix metalloproteinase-2, cluster of differentiation 44 and Ki-67, and increased that of the tumor suppressor, mothers against decapentaplegic homolog 2. Collectively, the findings of the present study indicated that miR-106b may target LAR4B to inhibit cancer cell viability, migration and invasion, and may be considered as a novel therapeutic target in PCa.
\end{abstract}

\section{Introduction}

Prostate cancer $(\mathrm{PCa})$ is the most common malignancy among males in Europe and USA, with increasing incidence (1).

Correspondence to: Dr Dongxu Zhang, Department of Urology, Ningbo First Hospital, 59 Liuting Street, Ningbo, Zhejiang 315010, P.R. China

E-mail: weiqi.yin@yandex.com

Key words: miR-106b, prostate cancer, la-related protein 4B, viability, migration, invasion
Additionally, $1 / 7$ of Australian males are diagnosed with PCa annually, at a mean age of 75 years; PCa is considered to be the second most fatal malignancy among the European and American male populations (2). In China, with the improvement of the living standards, changes in diet composition and the progressive aging of the population, the incidence of malignant tumors has notably increased; $\mathrm{PCa}$ in particular is quickly becoming the leading cause of mortality among middle-aged and elderly men $(3,4)$. At present, the main methods for diagnosing PCa are digital rectal examination (DRE), measurement of serum prostate-specific antigen (PSA) levels, magnetic resonance imaging and transrectal ultrasound-guided prostatic biopsy (5-8). In addition, DRE combined with PSA is considered as the standard method for early screening of PCa (6); however, the findings on DRE may be subjective, and the value of DRE in the diagnosis of early PCa without notable nodules is limited. On the contrary, PSA detection is characterized by high sensitivity and relatively low specificity, and the detection rate of PCa is only $25 \%$, particularly when PSA is between 4-10 ng/ml (PSA diagnostic gray zone) (9). Furthermore, PSA detection often leads to inaccurate diagnoses and poor therapeutic strategies for the treatment of PCa (10-12). In addition, the exact molecular mechanism underlying the development and progression of PCa remains unclear. Therefore, elucidating this mechanism is crucial for the clinical diagnosis, treatment and follow-up monitoring of patients with PCa.

MicroRNAs (miRNAs/miRs) are noncoding RNAs comprising $\sim 20-22$ nucleotides in length and are highly conserved among species, which act as key factors in tumor inhibition or promotion via the regulation of oncogenes or tumor suppressors (13). The association between miRNAs and the development, diagnosis and treatment of PCa has attracted notable attention (14-16). For example, miR-206 was reported to exert antitumor effects on PCa through regulating Annexin A2 and C-X-C motif chemokine $11(17,18)$; miR-33a acts as a tumor suppressor and was observed to be downregulated in PCa (19). Furthermore, miR-605 (20), miR-106a (21) and miR-483-5p (22) act as oncogenes in PCa. Conversely, miR-154 (23), miR-331-3p (24) and miR-625 (25) act as tumor suppressors in PCa. miR-106b is a member of the miR-106b-25 family that is highly expressed in laryngeal, gastric, breast and hepatic cancer, and has important functions in regulating tumor cell migration, invasion and proliferation (26-29). Furthermore, it was previously reported that miR-106b induces apoptosis and inhibits invasion of thyroid 
cancer cells by downregulating the expression of chromosome 1 open reading frame 24, suppresses the ability of Smad7 to enhance epithelial-to-mesenchymal transition and promotes the metastasis of esophageal cancer cells; inhibition of miR-106b induces apoptosis, and suppresses proliferation and migration of renal cell carcinoma cells (30-32). However, the role and the underlying molecular mechanisms of miR-106b in $\mathrm{PCa}$ require further investigation.

The aim of the present study was to investigate the effects of miR-106b on PCa cell viability, migration and invasion and the underlying mechanism of action, in order to determine whether PCa may be a novel biomarker for the diagnosis and treatment of PCa.

\section{Materials and methods}

Human tissue samples. The present study was approved by the Ethics Committee of Ningbo First Hospital (Ningbo, China). A total of 40 patients with PCa from Ningbo First Hospital were investigated, none of whom had received radiotherapy, chemotherapy or immunotherapy prior to tumor resection. All the patients provided written informed consent for their tissues to be used for research purposes.

Cell culture and transfection. The human PCa cell line LNCaP was purchased from the American Type Culture Collection (ATCC, Manassas, VA, USA), and routinely cultured in RPMI-1640 (Invitrogen; Thermo Fisher Scientific, Inc., Waltham, MA, USA) supplemented with $10 \%$ fetal bovine serum (FBS; Invitrogen; Thermo Fisher Scientific, Inc.) and $1 \%$ penicillin/streptomycin (Gibco; Thermo Fisher Scientific, Inc.) at $37^{\circ} \mathrm{C}$ in $5 \% \mathrm{CO}_{2}$.

miR-106 inhibitors, miR-negative control (NC) inhibitor, miR-106 mimics and miR-NC mimics were purchased from Ambion (Thermo Fisher Scientific, Inc.). Inhibitors and mimics were transfected at a concentration of $100 \mathrm{nM}$. LNCaP cells were seeded into 6-well plates and transfected with $100 \mathrm{nM}$ of miR-106 inhibitor, miR-NC inhibitor, miR-106 mimics and miR-NC mimics using Lipofectamine ${ }^{\circledR} 2000$ reagent (Invitrogen; Thermo Fisher Scientific, Inc.) according to the manufacturer's protocols for $48 \mathrm{~h}$. The sequences of miR-106 inhibitor, miR-NC inhibitor, miR-106 mimics and miR-NC mimics were as follows: miR-106b mimics, 5'-TAAAGTGCT GACAGTGCAGAT-3'; miR-NC mimics, 5'-UUCUCCGAA CGUGUCACGUTT-3'; miR-106b inhibitor, 5'-AUCUGCUCA GCACUUUA-3'; miR-NC inhibitor, 5'-CAGUACUUUUGU GUAGUACAA-3'.

Target prediction and luciferase assay. The prediction of the 3'-untranslated regions (3'-UTRs) of la-related protein 4B (LAR4B) as a binding target of miR-106b was checked by using TargetScan (version 7.1; www.targetscan.org/vert_71/). Subsequently, the 3'-UTR of LAR4B was mutated using a mutagenesis kit (Promega Corporation, Madison, WI, USA) according to the manufacturer's protocol. After transfection, cells were maintained at $37^{\circ} \mathrm{C}$ in $5 \% \mathrm{CO}_{2}$ for $48 \mathrm{~h}$. Wild-type (WT) and mutant (Mut) sequences of LAR4B were amplified and inserted into the pmirGLO vector (Shanghai GenePharma Co., Ltd., Shanghai, China) to construct luciferase reporter plasmids according to the manufacturer's protocol
(Promega Corporation). Cells were transfected with miR-106 mimics or miR-NC mimics and LAR4B 3'-UTR WT or LAR4B 3'-UTR Mut plasmids. Cells were maintained at $37^{\circ} \mathrm{C}$ in $5 \% \mathrm{CO}_{2}$ for $48 \mathrm{~h}$ after transfection. Luciferase activity was detected with a dual luciferase reporter kit (Promega Corporation). Renilla luciferase was used to normalize the luciferase activity.

Cell viability assay. Cells at a density of $5 \times 10^{3}$ cells/well were seeded in 96-well plates and transfected. After incubation for $0,12,24$ and $48 \mathrm{~h}$, the transfected cells were treated with $0.5 \mathrm{mg} / \mathrm{ml} \mathrm{MTT}$ solution and incubated in the dark at $37^{\circ} \mathrm{C}$ for $4 \mathrm{~h}$. Subsequently, the supernatants were removed and dimethyl sulfoxide was added to dissolve the formazan crystals. Then, the optical density at $490 \mathrm{~nm}$ was recorded using a microplate spectrophotometer. The experiment was performed in triplicate.

Wound healing assay. Cells at density of $5 \times 10^{5}$ cells/well were seeded into a 6-well plate. After cells attained $90 \%$ confluence, the cell monolayer was scratched using a $10-\mu 1$ pipette tip, and the cells were cultured in a serum-free DMEM (Thermo Fisher Scientific, Inc.) for cell recovery. Subsequently, the cells were imaged at $48 \mathrm{~h}$ with an inverted light microscope (Olympus, Tokyo, Japan; magnification, x200) and samples were observed in five randomly-selected fields of view.

Transwell invasion assay. Matrigel was diluted with serum-free medium (1:3) and then added to the upper chambers $(50 \mu 1$ per well) and allowed to form a gel for $30 \mathrm{~min}$ at $37^{\circ} \mathrm{C}$ with $5 \% \mathrm{CO}_{2}$. Then, transfected LNCaP cells $\left(1 \times 10^{6}\right.$ cells/well $)$ were seeded into the upper chamber with serum-free RPMI-1640 medium, whereas RPMI-1640 supplemented with 10\% FBS was added to the lower chamber. After incubation for $48 \mathrm{~h}$ in $5 \% \mathrm{CO}_{2}$ at $37^{\circ} \mathrm{C}$, the cells on the top of membranes were removed and the invading cells were fixed with $70 \%$ ethanol at room temperature for $30 \mathrm{~min}$ and stained with $0.5 \%$ crystal violet solution at room temperature for $30 \mathrm{~min}$, and counted using an with an inverted light microscope (magnification, x200) and samples were observed in five randomly-selected fields of view.

Reverse transcription-quantitative polymerase chain reaction (RT-qPCR) analysis. Total RNA from PCa tissues and cell lines was extracted with TRIzol reagent (Invitrogen; Thermo Fisher Scientific, Inc.), and miRNA was extracted using the miRcute miRNA Isolation kit (Tiangen, Shanghai, China). TaqMan MicroRNA Reverse Transcription kit and Taqman High-capacity cDNA kit (Applied Biosystems; Thermo Fisher Scientific, Inc.) were used to reverse transcribe miRNA and mRNA, respectively. The RT conditions were the following: $5 \mathrm{~min}$ at $25^{\circ} \mathrm{C}, 30 \mathrm{~min}$ at $42^{\circ} \mathrm{C}$ and $5 \mathrm{~min}$ at $85^{\circ} \mathrm{C}$. The expression of miR-106b was determined by RT-qPCR using the TaqMan miR kit (Applied Biosystems; Thermo Fisher Scientific, Inc.); the mRNA expression of LAR4B, matrix metalloproteinase-2 (MMP2), mothers against decapentaplegic homolog 2 (Smad2), cluster of differentiation (CD)44 and Ki-67 was measured using a TaqMan RT-qPCR kit (Applied Biosystems; Thermo Fisher Scientific, Inc.). The thermocycling conditions were the following: Initial denaturation at $95^{\circ} \mathrm{C}$ for $3 \mathrm{~min}$, followed by 40 cycles of $95^{\circ} \mathrm{C}$ for $15 \mathrm{sec}$ and $60^{\circ} \mathrm{C}$ for $60 \mathrm{sec}$. U6 and GAPDH 
Table I. Expression of miR-106b in the tissues of patients with PCa.

\begin{tabular}{|c|c|c|c|}
\hline Factors & Case & miR-106b (mean) & $\mathrm{P}$-value \\
\hline Age, years & & & 0.631 \\
\hline$\geqq 60$ & 22 & $2.164 \pm 0.112$ & \\
\hline$<60$ & 18 & $2.248 \pm 0.135$ & \\
\hline Serum PSA, ng/ml & & & 0.668 \\
\hline$\geqq 10$ & 23 & $2.225 \pm 0.139$ & \\
\hline$<10$ & 17 & $2.148 \pm 0.087$ & \\
\hline pT Stage & & & $0.007^{\mathrm{a}}$ \\
\hline$\geqq \mathrm{T} 3$ & 25 & $2.462 \pm 0.132$ & \\
\hline$<\mathrm{T} 3$ & 15 & $1.943 \pm 0.078$ & \\
\hline Histological grade & & & $0.028^{\mathrm{a}}$ \\
\hline $\begin{array}{l}\text { Well-intermediate } \\
\text { differentiation }\end{array}$ & 10 & $1.861 \pm 0.104$ & \\
\hline Poor differentiation & 30 & $2.391 \pm 0.128$ & \\
\hline Metastasis & & & $0.019^{\mathrm{a}}$ \\
\hline No & 31 & $1.938 \pm 0.095$ & \\
\hline Yes & 9 & $2.399 \pm 0.116$ & \\
\hline
\end{tabular}

${ }^{a} \mathrm{P}<0.05$. miR, microRNA; PSA, prostate-specific antigen.

were used as controls for miRNA and mRNA, respectively. Data were acquired by using a HT-7900 TaqMan instrument (Applied Biosystems; Thermo Fisher Scientific, Inc.). The mRNA relative expression levels were calculated using the $2^{-\Delta \Delta \mathrm{Cq}}$ method (33). The PCR primers were as follows: miR-106b, 5'-TTTTCGCCCTTAGCGTGAAGA-3' (forward) and 5'-GAGGCAGTCGAAGCTCTCG-3' (reverse); U6, 5'-CTCGCTTCGGCAGCACA-3' (forward) and 5'-AAC GCTTCACGAATTTGCGT-3' (reverse); LARP4B, 5'-TGG TCCTATATCGCAAACCACT-3' (forward) and 5'-GCACTA CTCGCTTCCAAATGT-3' (reverse); MMP2, 5'-GCTATG GACCTTGGGAGAA-3' (forward) and 5'-TGGAAGCGG AATGGAAAC-3' (reverse); Smad2, 5'-CATCAGCCAATG GCAAGTGAA-3' (forward) and 5'-AGAACAGGGTCTGCA TCCATCATA-3' (reverse); CD44, 5'-ACAACTGGTGAT GGAGACTCATCC-3' (forward) and 5'-CAGAGTGGCTTA TCATCTTGG-3' (reverse); and Ki-67, 5'-GCAGGACTT CACTTGCTTCC-3' (forward) and 5'-TCATTTGCGTTT GTTTCACG-3' (reverse); GAPDH, 5'-ACAACTTTGGTA TCGTGGAAGG-3' (forward); and 5'-GCCATCACGCCA CAGTTTC-3' (reverse).

Western blot analysis. Total protein from PCa tissues and LNCaP cells was extracted with radioimmunoprecipitation assay buffer (Beijing Solarbio Science \& Technology, Beijing, China), and the protein concentration was measured using the BCA Protein Assay kit (Vazyme, Piscataway, NJ, USA). Equal amounts of protein $(10 \mu \mathrm{g})$ were separated via $12 \%$ SDS-PAGE and transferred onto polyvinylidene difluoride membranes. The membranes were then blocked with $5 \%$ non-fat milk for $1 \mathrm{~h}$, followed by incubation at $4^{\circ} \mathrm{C}$ overnight with the following primary antibodies (1:1,000): LARP4B (cat. no. ab197085;

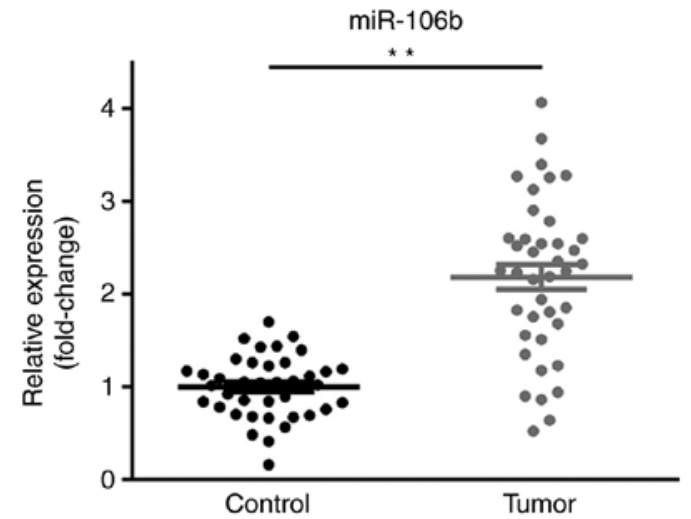

Figure 1. miR-106b is upregulated in patients with PCa. The expression of miR-106b in PCa tissues was measured by reverse transcription-quantitative polymerase chain reaction assay. The results are expressed as the mean \pm standard deviation of three independent experiments and each was performed in triplicate. ${ }^{* *} \mathrm{P}<0.01$ vs. adjacent normal tissues. miR, microRNA; PCa, prostate cancer.

Abcam, Cambridge, MA, USA), MMP2 (cat. no. 40994; Cell Signaling Technology, Inc.), Smad2 (cat. no. 8685; Cell Signaling Technology, Inc.), CD44 (cat. no. 37259; Cell Signaling Technology, Inc.), Ki-67 (cat. no. 4400; Cell Signaling Technology, Inc.) and GAPDH (cat. no. 5174; Cell Signaling Technology, Inc.). Subsequently, the membranes were washed with tris-buffered saline with $0.1 \%$ Tween-20 three times (15 min each time) and incubated with horseradish peroxidase-conjugated secondary antibodies $(1: 2,000)$ for a further $2 \mathrm{~h}$ at room temperature including anti-mouse (cat. no. 7076; Cell Signaling Technology, Inc.) and anti-rabbit (cat. no. 5127; Cell Signaling Technology, Inc.). Protein bands were examined via Quantity One software (version 4.5; Bio-Rad Laboratories, Inc., Hercules, CA, USA) with an ECL kit (Abcam). GAPDH was used as the loading control.

Statistical analysis. Each experiment was performed in triplicate. SPSS 19.0 software (IBM Corp., Armonk, NY, USA) was used to analyze the experimental data. The data were presented as the mean \pm standard deviation. Statistical differences between two groups were analyzed by a Student's t-test. Statistical differences among multiple groups were analyzed by one-way analysis of variance followed by a Bonferroni post-hoc test. $\mathrm{P}<0.05$ was considered to indicate a statistically significant difference.

\section{Results}

Clinicopathological features of the patients. As presented in Table I, the expression of miR-106b was significantly associated with pT stage, histological grade and lymphatic metastasis, but had no significant association with gender and age.

miR-106b is upregulated in patients with PCa. To evaluate the expression and clinical value of miR-106b in $\mathrm{PCa}, 40$ pairs of PCa tissues and matched adjacent normal tissues were collected and the expression of miR-106b was examined by RT-qPCR. The results demonstrated that the expression of miR-106b was significantly upregulated in PCa tissues relative to the respective adjacent normal tissues (Fig. 1). Therefore, 
A

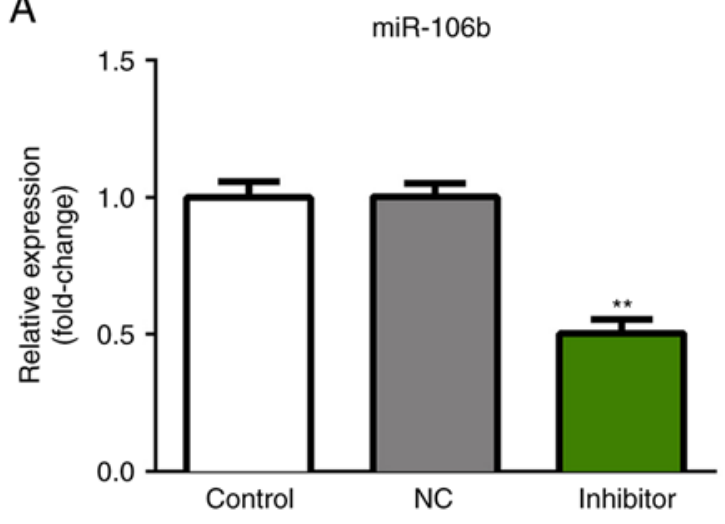

B

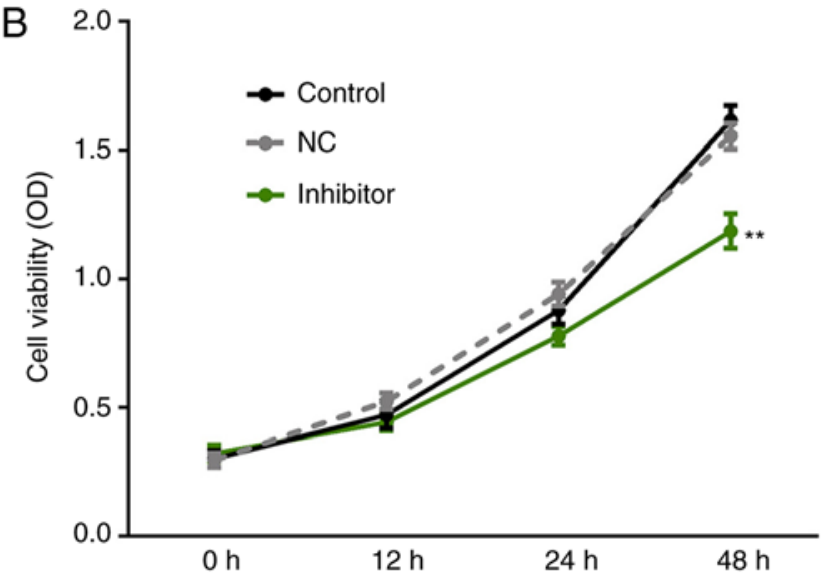

Figure 2. Downregulation of miR-106b suppresses prostate cancer cell viability. (A) Transfection efficiency was assessed by reverse transcription-quantitative polymerase chain reaction following transfection with miR-106b inhibitors in LNCaP cells. (B) Viabilities of LNCaP cells transfected with miR-106b inhibitors were assessed by MTT assay for $0,12,24$ and $48 \mathrm{~h}$. The results are expressed as the mean \pm standard deviation of three independent experiments and each was performed in triplicate. ${ }^{* *} \mathrm{P}<0.01$ vs. control group. miR, microRNA; NC, negative control.

miR-106b may have the potential as a novel biomarker for the diagnosis and prognosis of PCa.

Downregulation of miR-106b inhibits in PCa cell viability. To further investigate the role of miR-106b in the progression and development of PCa, miR-NC inhibitor and miR-106b inhibitor were respectively transfected into LNCaP cells, and RT-qPCR was performed to evaluate the transfection efficiency after $48 \mathrm{~h}$. The results demonstrated that the expression of miR-106b was significantly decreased relative to the negative control group in LNCaP cells (Fig. 2A). Subsequently, an MTT assay was conducted to evaluate the effects of miR-106b on the viability of LNCaP cells. As presented in Fig. 2B, inhibition of miR-106b exerted a significant inhibitory effect on the viability of LNCaP cells compared with the controls.

Inhibition of miR-106b suppresses the migration and invasion of PCa cells. In addition, to investigate the effects of miR-106b on the migration and invasion of PCa cells, wound healing and Transwell invasion assays, were respectively performed with LNCaP cells. The results revealed that knockdown of miR-106b significantly suppressed LNCaP cell migration after 48 h (Fig. 3A and B) compared with the control. Furthermore, the invasive ability of LNCaP cells was significantly decreased following transfection with miR-106b inhibitors for $48 \mathrm{~h}$ compared with the control (Fig. 3C and D). Collectively, these results indicate that the downregulation of miR-106b decreased the migration and invasive abilities of LNCaP cells.

Expression of miR-106b. As presented in Fig. 4, the expression of miR-106b was significantly increased following transfection with miR-106b mimics compared with the control; no significant difference between the control and miR-NC mimics groups was observed.

$L A R P 4 B$ is a direct target of $m i R-106 b$. To further characterize the possible downstream regulators of miR-106b affecting the development and progression of PCa, TargetScan was used to predict the target genes regulated by miR-106b. The results indicated that LARP4B is a potential target gene of miR-106b
(Fig. 5A). Furthermore, a luciferase reporter assay was used to further confirm whether miR-106b directly targets LARP4B in LNCaP cells. As presented in Fig. 5B, miR-106b could directly bind to the 3'-UTR of LARP4B after co-transfection of miR-106b mimic or miR-NC mimic, and the luciferase reporter vector. Upregulation of miR-106b significantly suppressed the luciferase activity of wild-type LARP4B 3'-UTR compared with the miR-NC group, but not that of mutated LARP4B 3'-UTR.

In addition, to further confirm whether LARP4B is a direct target gene of miR-106b, RT-qPCR and western blot analyses were performed to evaluate the mRNA and protein levels of LARP4B in PCa tissues and the matched adjacent normal tissues. As presented in Fig. 5C and D, the expression of LARP4B was decreased at the mRNA and protein level in PCa tissues compared with in the matched adjacent normal tissues.

Furthermore, the expression of LARP4B in LNCaP cells after transfection with miR-106b inhibitors was measured by RT-qPCR, respectively. The results revealed that miR-106b downregulation significantly increased the mRNA (Fig. 5E) and protein (Fig. 6E and F) expression of LARP4B compared with the control.

Knockdown of miR-106b regulates the expression of $M M P 2$, Smad2, CD44 and Ki-67. To further confirm the role of miR-106b in the progression and development of $\mathrm{PCa}$, the expression of proteins associated with the proliferation and metastasis of PCa, including MMP2, Smad2, CD44 and Ki-67, was measured by RT-qPCR analysis and western blotting. As presented in Fig. 6A-F, inhibition of miR-106b significantly suppressed the expression of MMP2, CD44 and Ki-67, whereas it markedly increased the expression of Smad 2 compared with the control.

\section{Discussion}

miR-106b was reported to be aberrantly expressed in numerous types of cancers, such as non-small cell lung cancer (34), breast cancer (35) and renal cell cancer (36). Additionally, miR-106b was differentially expressed in colorectal cancer (37), esophageal squamous cell carcinoma (38) and 
A

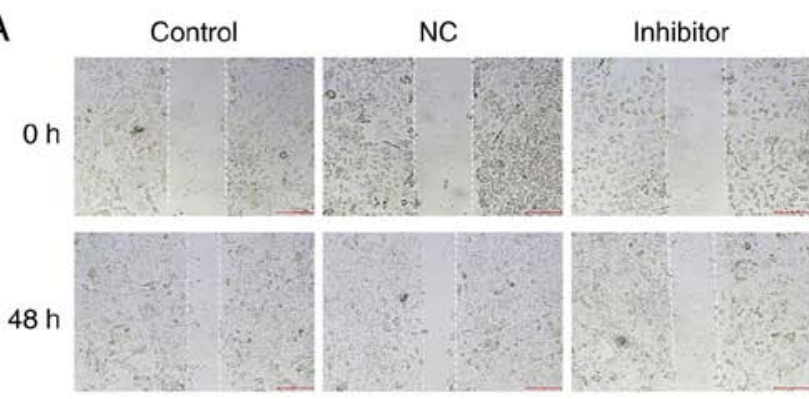

C

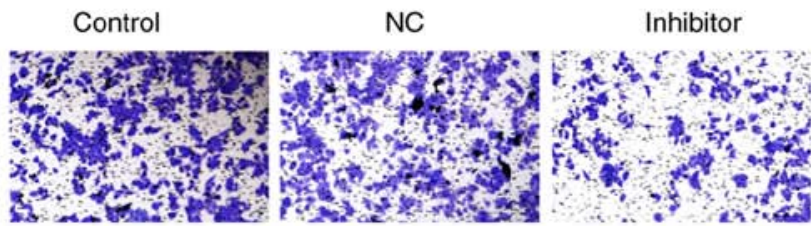

$\mathrm{B}$

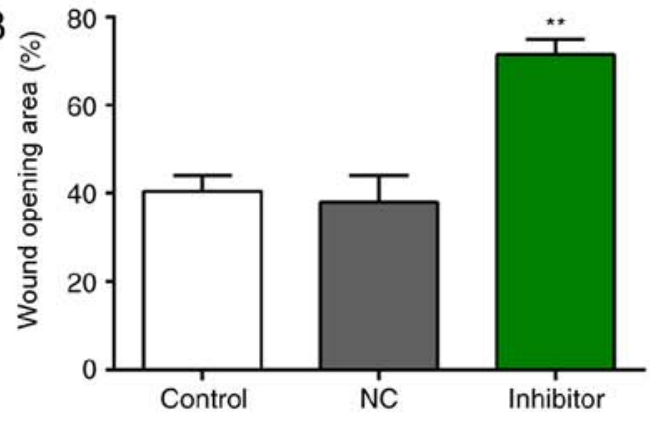

D

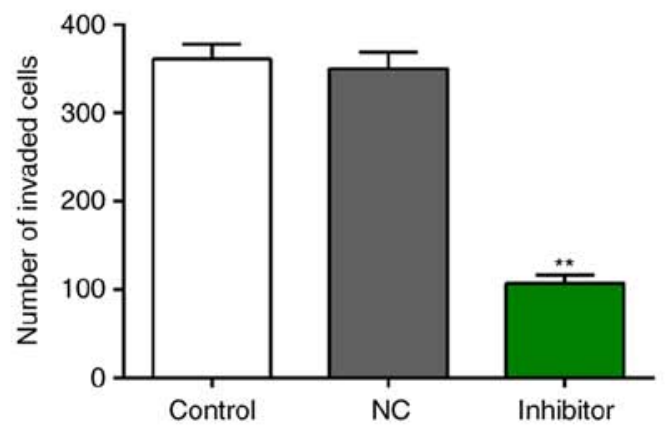

Figure 3. Inhibition of miR-106b suppresses the migration and invasion of prostate cancer cells. (A and B) The migration ability of LNCaP cells transfected with miR-106b inhibitors for $48 \mathrm{~h}$ was examined by a wound healing assay. (C and D) The invasive ability of LNCaP cells transfected with miR-106b inhibitors for $48 \mathrm{~h}$ was evaluated by a Transwell invasion assay. Magnification, $\mathrm{x} 200$. The results are expressed as the mean \pm standard deviation of three independent experiments and each was performed in triplicate. ${ }^{* *} \mathrm{P}<0.01$ vs. control group. $\mathrm{NC}$, negative control.



Figure 4. Expression of miR-106b. The expression of miR-106b in cells treated with miR-106b mimics was significantly higher than that in NC. The difference between control group and NC group was of no statistical significance. ${ }^{* *} \mathrm{P}<0.01$ vs. NC group. miR, microRNA; NC, negative control.

hepatocellular cancer (39). miR-106b was downregulated in thyroid cancer tissues, while overexpressed miR-106b inhibited the migration and invasion of thyroid cancer cells (30); however, miR-106b was upregulated in gastric cancer, and downregulated miR-106b reduced the migration and invasion of gastric cancer cells (27). Therefore, miR-106b may serve an oncogenic or anti-tumor role in cancer. However, the possible roles of miR-106b in prostate cancer require further investigation.

In the present study, miR-106b was overexpressed in $\mathrm{PCa}$ tissues. The expression of miR-106b in PCa cells was significantly decreased following treatment with miR-106b inhibitor. We proposed that miR-106b may act as oncogene in PCa. Previous studies showed that miR-106b was upregulated in $\mathrm{PCa}$, and was associated with the progression and prognosis of PCa, suggesting that miR-106b could serve as novel clinical markers of PCa $(40,41)$. However, the underlying mechanisms remain unclear.

LARPs are a class of RNA-binding proteins that are located at different subcellular sites and interact with RNA, serving an important role in cell transcription and translation $(42,43)$. In recent years, LARPs have been reported to serve important roles in the proliferation, differentiation, migration and angiogenesis of several malignant tumors (44). LARP4B, a member of the LARP family, acts as a tumor suppressor in glioma (45); however, its expression and regulatory mechanism in $\mathrm{PCa}$ have not been elucidated. In the present study, we determined that the expression of LARP4B was downregulated in $\mathrm{PCa}$ tissues. Furthermore, LARP4B was reported as a target gene of miR-106b. Additionally, downregulation of miR-106b increased the expression of LARP4B. These results support LARP4B as a critical downstream mediator of miR-106b, involved in the progression and development of PCa.

We further investigated the potential roles of miR-106b on the behavior of PCa cells. Downregulated miR-106b suppressed the cell viability, migration and invasion of $\mathrm{PCa}$ cells. In addition, knockdown of miR-106 downregulated the expression of MMP2, Ki67 and CD44, but increased that of Smad2. Upregulated MMP2 promoted the migration and invasion of glioblastoma multiforme cells (46). Mutations or deletions in Smad2 can interrupt transforming growth factor (TGF)- $\beta$ signal transduction, and lead to reduced growth inhibition of induced by TGF- $\beta$, resulting in tumor development (47-49). CD44 is highly expressed in malignant tumor cells and its expression is closely associated with patient prognosis (50-52). miR-200b-3p regulated the proliferation and apoptosis of colorectal cancer cells, and inhibited Ki-67 signaling (53). MMP2, Smad2, Ki-67 and CD44 serve an 


\section{A Position 65-72 of LARP4B 3'UTR 5' ...AGACACUCUCCCACUCAGUGCGA...}

1111111

hsa-miR-106b $\quad 3^{\prime}$ CGUCGUUCAUGGGUGUCACGCC
B

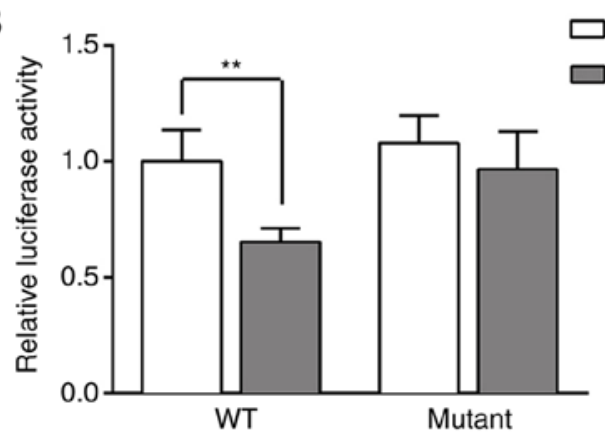

D

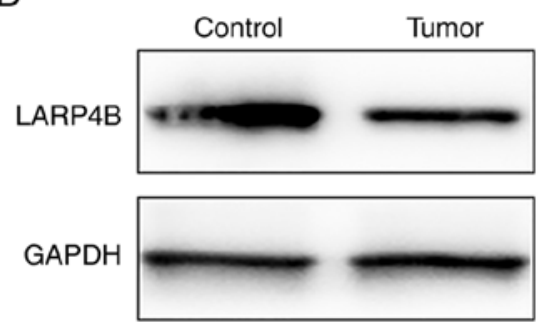

C

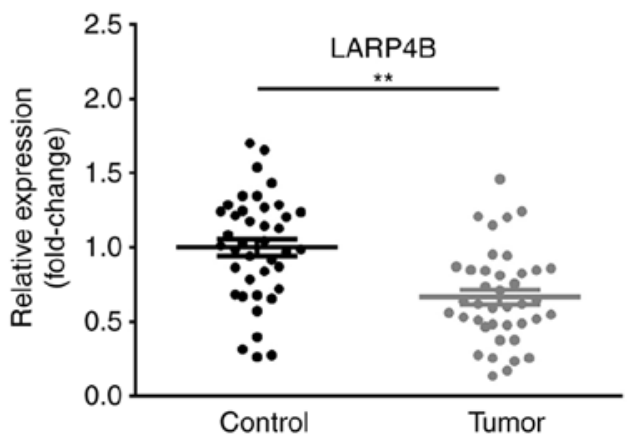

LARP4B

E



Figure 5. LARP4B is a direct target of miR-106b. (A) The prediction of the binding between miR-106b and LARP4B was performed using TargetScan. (B) Luciferase reporter assays were performed to verify the binding of miR-106b to the 3'-UTR of LARP4B in cells treated with miR-106b mimic or miR-NC mimic. (C) The mRNA and (D) protein expression levels of LARP4B in PCa tissues were examined by RT-qPCR and western blot assays. (E) The mRNA expression levels of LARP4B in LNCaP cells transfected with miR-106b inhibitor or miR-NC inhibitor after transfection with miR-106b was examined by RT-qPCR. The band intensity was quantified by ImageJ software. The results are expressed as the mean \pm standard deviation of three independent experiments and each was performed in triplicate. ${ }^{* *} \mathrm{P}<0.01$ vs. control group. hsa, homo sapiens; LARP4b, la-related protein 4B; PCa, prostate cancer; miR, microRNA; $\mathrm{NC}$, negative control; RT-qPCR, reverse transcription-quantitative polymerase chain reaction; UTR, untranslated region; WT, wild-type.

A
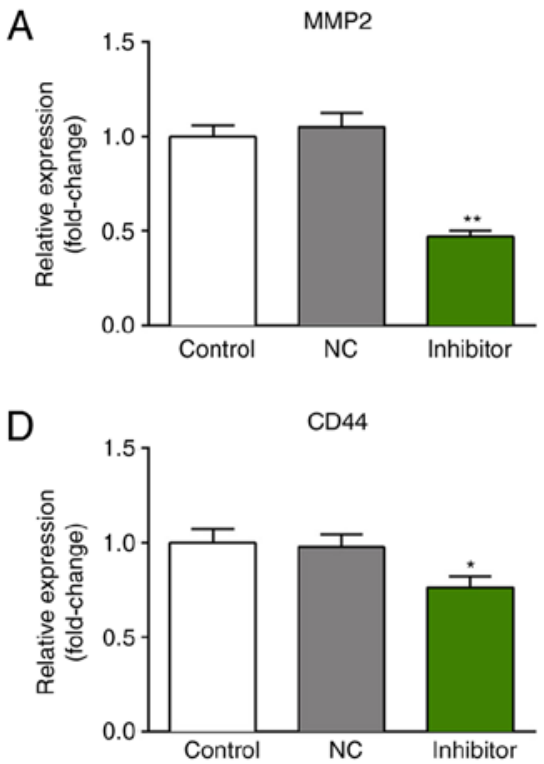

B

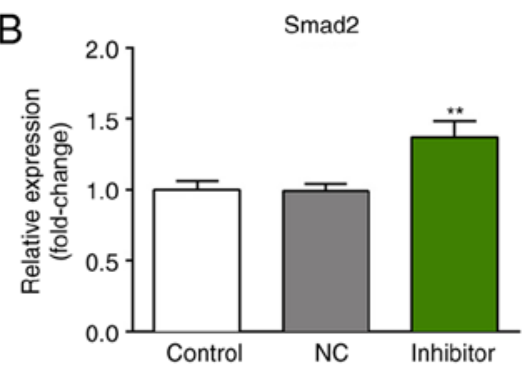

$\mathrm{E}$

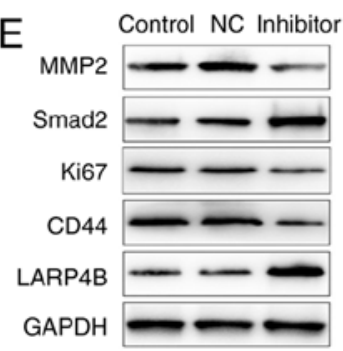

$\mathrm{F}$
C
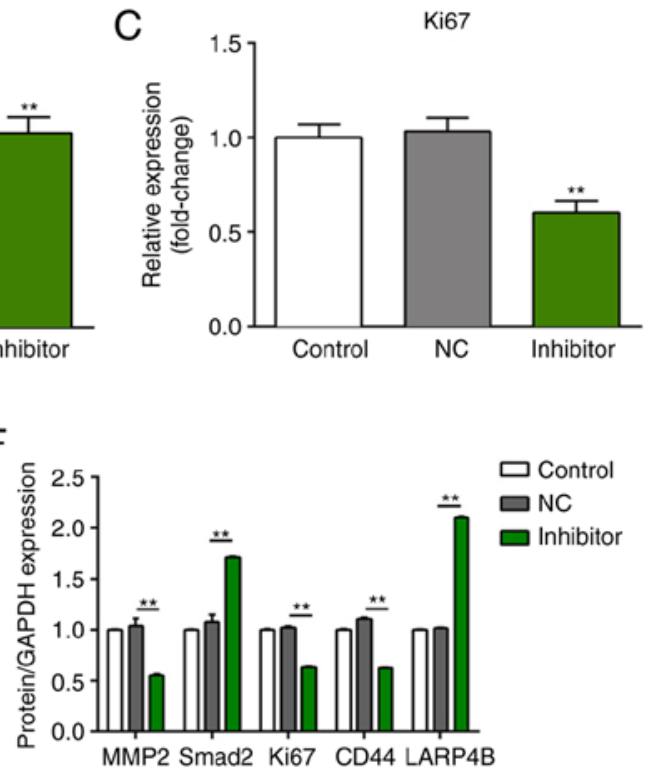

Figure 6. Knockdown of miR-106b regulates the expression of MMP2, Smad2, CD44 and Ki-67. (A-D) The mRNA and (E and F) protein expression levels of MMP2, Smad2, Ki-67 and CD44 in LNCaP cells after transfection with miR-106b, which were examined by reverse transcription-quantitative polymerase chain reaction and western blot analysis, repectively. The band intensity was quantified by ImageJ software. The results are expressed as the mean \pm standard deviation of three independent experiments and each was performed in triplicate. ${ }^{*} \mathrm{P}<0.05,{ }^{* *} \mathrm{P}<0.01$ vs. control group. $\mathrm{CD}$, cluster of differentiation; MMP, matrix metalloproteinase; Smad2, mothers against decapentaplegic homolog 2 . 
important role in the proliferation, migration and invasion of tumor cells (54-56). The role of miR-106b in regulating the expression of MMP2, Smad2, Ki-67 and CD44 further suggested that miR-106b could suppress the viability, migration, and invasion of PCa cells.

In conclusion, the present study demonstrated that miR-106b targets LAR4B to suppress cancer cell viability, migration and invasion; thus; miR-106b may represent a novel target for the treatment of patients with PCa. However, there was a limitation in the present study, in which no evidence was provided to link LARP4B exclusively to miR-106b and its function in cancer, which will be further investigated in the future.

\section{Acknowledgements}

Not applicable.

\section{Funding}

No funding was received.

\section{Availability of data and materials}

The datasets used and/or analyzed during the current study are available from the corresponding author on reasonable request.

\section{Authors' contributions}

WY drafted the manuscript. WY, JC and GW collected, analyzed and interpreted the data. DZ conceived and designed the present study.

\section{Ethics approval and consent to participate}

The present study was approved by the Ethics Committee of Ningbo First Hospital (Ningbo, China). Patients provided written informed cosnent.

\section{Patient consent for publication}

Not applicable.

\section{Competing interests}

The authors declare that they have no competing interests.

\section{References}

1. Siegel RL, Miller KD and Jemal A: Cancer statistics, 2018. CA Cancer J Clin 68: 7-30, 2018

2. Ferlay J, Colombet M, Soerjomataram I, Dyba T, Randi G, Bettio M, Gavin A, Visser O and Bray F: Cancer incidence and mortality patterns in Europe: Estimates for 40 countries and 25 major cancers in 2018. Eur J Cancer 103: 356-387, 2018.

3. Chen W, Zheng R, Baade PD, Zhang S, Zeng H, Bray F, Jemal A, Yu XQ and He J: Cancer statistics in China, 2015. CA Cancer J Clin 66: 115-132, 2016.

4. Ren SC, Chen R and Sun YH: Prostate cancer research in China. Asian J Androl 15: 350-353, 2013.

5. Naji L, RandhawaH, Sohani Z, Dennis B,Lautenbach D, Kavanagh O, Bawor M, Banfield L and Profetto J: Digital rectal examination for prostate cancer screening in primary care: A systematic review and meta-analysis. Ann Fam Med 16: 149-154, 2018.
6. Liss MA, Chen H, Hemal S, Krane S, Kane CJ, Xu J and Kader AK: Impact of family history on prostate cancer mortality in white men undergoing prostate specific antigen based screening. J Urol 193: 75-79, 2015.

7. Durmus T, Baur A and Hamm B: Multiparametric magnetic resonance imaging in the detection of prostate cancer. Rofo 186: 238-246, 2014.

8. Boesen L, Nørgaard N, Løgager V, Balslev I and Thomsen HS: A prospective comparison of selective multiparametric magnetic resonance imaging fusion-targeted and systematic transrectal ultrasound-guided biopsies for detecting prostate cancer in men undergoing repeated biopsies. Urol Int 99: 384-391, 2017.

9. Moghul M, Somani B, Lane T, Vasdev N, Chaplin B, Peedell C, KandaSwamy GV and Rai BP: Detection rates of recurrent prostate cancer: ${ }^{68} \mathrm{Gallium}(\mathrm{Ga})$-labelled prostate-specific membrane antigen versus choline PET/CT scans. A systematic review. Ther Adv Urol 11: 1756287218815793, 2019.

10. Teoh JY, Yuen SK, Tsu JH, Wong CK, Ho BSh, Ng AT, Ma WK, Ho KL and Yiu MK: Prostate cancer detection upon transrectal ultrasound-guided biopsy in relation to digital rectal examination and prostate-specific antigen level: What to expect in the Chinese population? Asian J Androl 17: 821-825, 2015.

11. Javali TD, Dwivedi DK, Kumar R, Jagannathan NR, Thulkar S and Dinda AK: Magnetic resonance spectroscopy imaging-directed transrectal ultrasound biopsy increases prostate cancer detection in men with prostate-specific antigen between $4-10 \mathrm{ng} / \mathrm{ml}$ and normal digital rectal examination. Int J Urol 21: 257-262, 2014.

12. Kash DP, Lal M, Hashmi AH and Mubarak M: Utility of digital rectal examination, serum prostate specific antigen, and transrectal ultrasound in the detection of prostate cancer: A developing country perspective. Asian Pac J Cancer Prev 15: 3087-3091, 2014

13. McCann JV, Xiao L, Kim DJ, Khan OF, Kowalski PS, Anderson DG, Pecot CV, Azam SH, Parker JS, Tsai YS, et al: Endothelial miR-30c suppresses tumor growth via inhibition of TGF- $\beta$-induced Serpine1. J Clin Invest 130: 1654-1670, 2019.

14. Lee J, Kwon MH, Kim JA and Rhee WJ: Detection of exosome miRNAs using molecular beacons for diagnosing prostate cancer. Artif Cells Nanomed Biotechnol 46 (Suppl 3): S52-S63, 2018.

15. Malla B, Aebersold DM and Dal Pra A: Protocol for serum exosomal miRNAs analysis in prostate cancer patients treated with radiotherapy. J Transl Med 16: 223, 2018.

16. Xiaoli Z, Yawei W, Lianna L, Haifeng L and Hui Z: Screening of target genes and regulatory function of miRNAs as prognostic indicators for prostate cancer. Med Sci Monit 21: 3748-3759, 2015.

17. Yang N, Wang L, Liu J, Liu L, Huang J, Chen X and Luo Z: MicroRNA-206 regulates the epithelial-mesenchymal transition and inhibits the invasion and metastasis of prostate cancer cells by targeting Annexin A2. Oncol Lett 15: 8295-8302, 2018.

18. Wang Y, Xu H, Si L, Li Q, Zhu X, Yu T and Gang X: MiR-206 inhibits proliferation and migration of prostate cancer cells by targeting CXCL11. Prostate 78: 479-490, 2018.

19. Karatas OF, Wang J, Shao L, Ozen M, Zhang Y, Creighton CJ and Ittmann M: miR-33a is a tumor suppressor microRNA that is decreased in prostate cancer. Oncotarget 8: 60243-60256, 2017.

20. Zhou YJ, Yang HQ, Xia W, Cui L, Xu RF, Lu H, Xue Z, Zhang B, Tian ZN, Cao YJ, et al: Down-regulation of miR-605 promotes the proliferation and invasion of prostate cancer cells by up-regulating EN2. Life Sci 190: 7-14, 2017.

21. Luo B, Kang N, Chen Y, Liu L and Zhang Y: Oncogene miR-106a promotes proliferation and metastasis of prostate cancer cells by directly targeting PTEN in vivo and in vitro. Minerva Med 109: 24-30, 2018.

22. Yang ZG, Ma XD, He ZH and Guo YX: miR-483-5p promotes prostate cancer cell proliferation and invasion by targeting RBM5. Int Braz J Urol 43: 1060-1067, 2017.

23. Zhu C, Li J, Cheng G, Zhou H, Tao L, Cai H, Li P, Cao Q, Ju X, Meng X, et al: miR-154 inhibits EMT by targeting HMGA2 in prostate cancer cells. Mol Cell Biochem 379: 69-75, 2013.

24. Epis MR, Giles KM, Beveridge DJ, Richardson KL, Candy PA, Stuart LM, Bentel J, Cohen RJ and Leedman PJ: miR-331-3p and Aurora Kinase inhibitor II co-treatment suppresses prostate cancer tumorigenesis and progression. Oncotarget 8: 55116-55134, 2017.

25. Jackson BL, Grabowska A and Ratan HL: MicroRNA in prostate cancer: Functional importance and potential as circulating biomarkers. BMC Cancer 14: 930, 2014.

26. Xu Y, Wang K, Gao W, Zhang C, Huang F, Wen S and Wang B: MicroRNA-106b regulates the tumor suppressor RUNX3 in laryngeal carcinoma cells. FEBS Lett 587: 3166-3174, 2013. 
27. Yang TS, Yang XH, Chen X, Wang XD, Hua J, Zhou DL, Zhou B and Song ZS: MicroRNA-106b in cancer-associated fibroblasts from gastric cancer promotes cell migration and invasion by targeting PTEN. FEBS Lett 588: 2162-2169, 2014.

28. Smith AL, Iwanaga R, Drasin DJ, Micalizzi DS, Vartuli RL, Tan AC and Ford HL: The miR-106b-25 cluster targets Smad7, activates TGF- $\beta$ signaling, and induces EMT and tumor initiating cell characteristics downstream of Six1 in human breast cancer. Oncogene 31: 5162-5171, 2012.

29. Li Y, Tan W, Neo TW, Aung MO, Wasser S, Lim SG and Tan TM: Role of the miR-106b-25 microRNA cluster in hepatocellular carcinoma. Cancer Sci 100: 1234-1242, 2009.

30. Carvalheira G, Nozima BH and Cerutti JM: microRNA-106b-mediated down-regulation of $\mathrm{C} 1$ orf 24 expression induces apoptosis and suppresses invasion of thyroid cancer. Oncotarget 6: 28357-28370, 2015.

31. Dai F, Liu T, Zheng S, Liu Q, Yang C, Zhou J, Chen Y, Sheyhidin I and Lu X: MiR-106b promotes migration and invasion through enhancing EMT via downregulation of Smad 7 in Kazakh's esophageal squamous cell carcinoma. Tumour Biol 37 : 14595-14604, 2016.

32. Li Y, Chen D, Su Z, Li Y, Liu J, Jin L, Shi M, Jiang Z, Qi Z, Gui Y, et al: MicroRNA-106b functions as an oncogene in renal cell carcinoma by affecting cell proliferation, migration and apoptosis. Mol Med Rep 13: 1420-1426, 2016.

33. Livak KJ and Schmittgen TD: Analysis of relative gene expression data using real-time quantitative PCR and the 2(-Delta Delta C(T)) method. Methods 25: 402-408, 2001

34. Wei K, Pan C, Yao G, Liu B, Ma T, Xia Y, Jiang W, Chen L and Chen Y: miR-106b-5p promotes proliferation and inhibits apoptosis by regulating BTG3 in non-small cell lung cancer. Cell Physiol Biochem 44: 1545-1558, 2017.

35. Li N, Miao Y, Shan Y, Liu B, Li Y, Zhao L and Jia L: miR-106b and miR-93 regulate cell progression by suppression of PTEN via PI3K/Akt pathway in breast cancer. Cell Death Dis 8: e2796, 2017.

36. Lu J, Wei JH, Feng ZH, Chen ZH, Wang YQ, Huang Y, Fang Y, Liang YP, Cen JJ, Pan YH, et al: miR-106b-5p promotes renal cell carcinoma aggressiveness and stem-cell-like phenotype by activating Wnt/3-catenin signalling. Oncotarget 8: 21461-21471, 2017.

37. Ni S, Weng W, Xu M, Wang Q, Tan C, Sun H, Wang L, Huang D, Du $X$ and Sheng W: miR-106b-5p inhibits the invasion and metastasis of colorectal cancer by targeting CTSA. Onco Targets Ther 11: 3835-3845, 2018.

38. Zhang J, Chen D, Liang S, Wang J, Liu C, Nie C, Shan Z, Wang L, Fan Q and Wang F: miR-106b promotes cell invasion and metastasis via PTEN mediated EMT in ESCC. Oncol Lett 15: 4619-4626, 2018

39. Yen CS, Su ZR, Lee YP, Liu IT and Yen CJ: miR-106b promotes cancer progression in hepatitis B virus-associated hepatocellular carcinoma. World J Gastroenterol 22: 5183-5192, 2016.

40. Liang H, Studach L, Hullinger RL, Xie J and Andrisani OM: Down-regulation of RE-1 silencing transcription factor (REST) in advanced prostate cancer by hypoxia-induced miR-106b 25. Exp Cell Res 320: 188-199, 2014

41. Hudson RS, Yi M, Esposito D, Glynn SA, Starks AM, Yang Y, Schetter AJ, Watkins SK, Hurwitz AA, Dorsey TH, et al: MicroRNA-106b-25 cluster expression is associated with early disease recurrence and targets caspase- 7 and focal adhesion in human prostate cancer. Oncogene 32: 4139-4147, 2013.
42. Merret R, Martino L, Bousquet-Antonelli C, Fneich S, Descombin J, Billey E, Conte MR and Deragon JM: The association of a La module with the PABP-interacting motif PAM2 is a recurrent evolutionary process that led to the neofunctionalization of La-related proteins. RNA 19: 36-50, 2013.

43. Hussain RH, Zawawi M and Bayfield MA: Conservation of RNA chaperone activity of the human La-related proteins 4, 6 and 7. Nucleic Acids Res 41: 8715-8725, 2013.

44. Stavraka C and Blagden S: The La-related proteins, a family with connections to cancer. Biomolecules 5: 2701-2722, 2015.

45. Koso H, Yi H, Sheridan P, Miyano S, Ino Y, Todo T and Watanabe S: Identification of RNA-binding protein LARP4B as a tumor suppressor in glioma. Cancer Res 76: 2254-2264, 2016.

46. Liu J, Yang J, Yu L, Rao C, Wang Q, Sun C, Shi C, Hua D, Zhou X, Luo W, et al: miR-361-5p inhibits glioma migration and invasion by targeting SND1. Onco Targets Ther 11: 5239-5252, 2018.

47. Bao Y, Chen Z, Guo Y, Feng Y, Li Z, Han W, Wang J, Zhao W, Jiao Y, Li K, et al: Tumor suppressor microRNA-27a in colorectal carcinogenesis and progression by targeting SGPP1 and Smad2. PLoS One 9: e105991, 2014.

48. Tu B, Peng ZX, Fan QM, Du L, Yan W and Tang TT: Osteosarcoma cells promote the production of pro-tumor cytokines in mesenchymal stem cells by inhibiting their osteogenic differentiation through the TGF- $\beta /$ Smad2/3 pathway. Exp Cell Res 320: 164-173, 2014.

49. Fleming NI, Jorissen RN, Mouradov D, Christie M, Sakthianandeswaren A, Palmieri M, Day F, Li S, Tsui C, Lipton L, et al: SMAD2, SMAD3 and SMAD4 mutations in colorectal cancer. Cancer Res 73: 725-735, 2013.

50. Mattheolabakis G, Milane L, Singh A and Amiji MM: Hyaluronic acid targeting of CD44 for cancer therapy: From receptor biology to nanomedicine. J Drug Target 23: 605-618, 2015.

51. Prochazka L, Tesarik R and Turanek J: Regulation of alternative splicing of CD44 in cancer. Cell Signal 26: 2234-2239, 2014.

52. Kinugasa Y, Matsui T and Takakura N: CD44 expressed on cancer-associated fibroblasts is a functional molecule supporting the stemness and drug resistance of malignant cancer cells in the tumor microenvironment. Stem Cells 32: 145-156, 2014.

53. Chen L, Wang X, Zhu Y, Zhu J and Lai Q: miR-200b-3p inhibits proliferation and induces apoptosis in colorectal cancer by targeting Wnt1. Mol Med Rep 18: 2571-2580, 2018.

54. Yadav L, Puri N, Rastogi V, Satpute P, Ahmad R and Kaur G: Matrix metalloproteinases and cancer-roles in threat and therapy. Asian Pac J Cancer Prev 15: 1085-1091, 2014.

55. Ok Atılgan A, Özdemir BH, Akçay EY, Ataol Demirkan Ö, Tekindal MA and Özkardeş H: Role of tumor-associated macrophages in the Hexim1 and TGF $\beta /$ SMAD pathway, and their influence on progression of prostatic adenocarcinoma. Pathol Res Pract 212: 83-92, 2016.

56. Lee IH, Sohn M, Lim HJ, Yoon S, Oh H, Shin S, Shin JH, Oh SH, Kim J, Lee DK, et al: Ahnak functions as a tumor suppressor via modulation of TGF $\beta /$ Smad signaling pathway. Oncogene 33: 4675-4684, 2014.

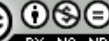

This work is licensed under a Creative Commons Attribution-NonCommercial-NoDerivatives 4.0 International (CC BY-NC-ND 4.0) License. 